\title{
Unconventional Superconductivity Induced by Suppressing an Iron-Selenium-Based Mott Insulator $\mathrm{CsFe}_{4-x} \mathrm{Se}_{4}$
}

\author{
Jin Siø, Guan-Yu Chen, Qing Li, Xiyu Zhu, Huan Yang, and Hai-Hu Wen ${ }^{*}$ \\ National Laboratory of Solid State Microstructures and Department of Physics, \\ Center for Superconducting Physics and Materials, Collaborative Innovation Center for Advanced \\ Microstructures, Nanjing University, Nanjing 210093, China
}

(Received 7 May 2020; revised 31 July 2020; accepted 11 August 2020; published 12 October 2020)

\begin{abstract}
There are several FeSe based superconductors, including the bulk FeSe, monolayer FeSe thin film, intercalated $\mathrm{K}_{x} \mathrm{Fe}_{2-y} \mathrm{Se}_{2}$ and $\mathrm{Li}_{1-x} \mathrm{Fe}_{x} \mathrm{OHFeSe}$, etc. Their normal states all show metallic behavior. The key player here is the FeSe layer, which exhibits the highest superconducting transition temperature in the form of monolayer thin film. Recently, a new FeSe based compound, $\mathrm{CsFe}_{4-x} \mathrm{Se}_{4}$, with the space group of $\mathrm{Bmmm}$ was found. Interestingly, the system shows a strong insulatorlike behavior, although it shares the same FeSe planes as other relatives. Density functional theory calculations indicate that it should be a metal, in sharp contrast with the experimental observations. Here, we report the emergence of unconventional superconductivity by applying pressure to suppress this insulatorlike behavior. At ambient pressure, the insulatorlike behavior cannot be modeled as a band insulator, but it can be described by the variable-range-hopping model for correlated systems. Furthermore, the specific heat down to $400 \mathrm{mK}$ has been measured, and a significant residual coefficient $\gamma_{0}=C /\left.T\right|_{T \rightarrow 0}$ is observed, which contrasts the insulatorlike state and suggests some quantum freedom of spin dynamics. By applying pressure, the insulatorlike behavior is gradually suppressed, and the system becomes a metal; finally, superconductivity is achieved at about $5.1 \mathrm{~K}$. The superconducting transition strongly depends on magnetic field and applied current, indicating a fragile superfluid density. Our results suggest that the superconductivity is established by diluted Cooper pairs on top of a strong correlation background in $\mathrm{CsFe}_{4-x} \mathrm{Se}_{4}$.
\end{abstract}

DOI: $10.1103 /$ PhysRevX.10.041008

Subject Areas: Condensed Matter Physics

\section{INTRODUCTION}

The iron-selenium (FeSe) based superconductors, which are different from the iron-arsenic based superconductors, have attracted a lot of attention recently because of their intriguing properties [1-19]. The FeSe has the simplest structure, and the critical temperature $\left(T_{c}\right)$ of the bulk samples [1] can be enhanced from about $8 \mathrm{~K}$ to $37 \mathrm{~K}$ by pressure [2-4]. The Meissner shielding signal was observed up to $65 \mathrm{~K}$ for $\mathrm{FeSe}$ monolayer thin film grown on a $\mathrm{Nb}$ doped $\mathrm{SrTiO}_{3}$ substrate [5-7], which could be the highest $T_{c}$ of all iron-based superconductors discovered so far (in terms of the Meissner effect). By intercalating alkali metals between FeSe layers, superconductivity was observed in a series of compounds, such as $\mathrm{A}_{x} \mathrm{Fe}_{2-y} \mathrm{Se}_{2}(\mathrm{~A}=\mathrm{Na}, \mathrm{K}, \mathrm{Rb}$, Cs, $\mathrm{Tl} / \mathrm{Rb}$, and $\mathrm{Tl} / \mathrm{K}$ ) [8-15] and $\mathrm{Li}_{1-x} \mathrm{Fe}_{x} \mathrm{OHFeSe}$ [16], and $T_{c}$ can be enhanced to $32 \mathrm{~K}$, or even to $46 \mathrm{~K}$. Also, the recently found hole-type $\mathrm{FeSe}$ based superconductor

\footnotetext{
*hhwen@nju.edu.cn
}

Published by the American Physical Society under the terms of the Creative Commons Attribution 4.0 International license. Further distribution of this work must maintain attribution to the author(s) and the published article's title, journal citation, and DOI.
$\mathrm{S}_{0.24}\left(\mathrm{NH}_{3}\right)_{0.26} \mathrm{Fe}_{2} \mathrm{Se}_{2}$ enriches the physics in the family of FeSe based superconductors [17]. An obvious common feature is that they all have FeSe layers as conducting sheets from which the superconductivity originates. The normal states of these systems show clear metallic behavior. Recently, a new FeSe based compound $\mathrm{CsFe}_{4-x} \mathrm{Se}_{4}$ was found [18]; it shows distinct features, although it has a roughly perfect FeSe layer. Unlike the system $\mathrm{A}_{x} \mathrm{Fe}_{2-y} \mathrm{Se}_{2}$ which has a clear phase separation, the $\mathrm{CsFe}_{4-x} \mathrm{Se}_{4}$ is uniform without the trace of phase separation. This compound shows an insulatorlike behavior and does not undergo clear antiferromagnetic transitions. At room temperature, it forms an orthorhombic lattice structure with a space group of $\mathrm{Bmmm}$; however, bulk FeSe undergoes a structural transition from tetragonal to orthorhombic at around $90 \mathrm{~K}$ $[2,3,19]$. The density functional theory (DFT) calculations on $\mathrm{CsFe}_{4-x} \mathrm{Se}_{4}$ indicate that it should be a metal with an intermediate density of states (DOS) [18] at the Fermi level compared with the value of $\mathrm{FeSe}$ and $\mathrm{A}_{x} \mathrm{Fe}_{2-y} \mathrm{Se}_{2}$, which is in sharp contrast with the experimentally observed insulatorlike behavior. The insulatorlike behavior in $\mathrm{CsFe}_{4-x} \mathrm{Se}_{4}$ remains puzzling and elusive.

In this paper, we report the successful synthesis of this new compound with high-quality and measurements of its intriguing features. At ambient pressure, it behaves as an 
insulator, and the resistivity obeys the relation $\ln \rho \propto$ $(1 / T)^{1 / 4}$ in a wide temperature range, which is consistent with the prediction of the variable-range-hopping (VRH) model for correlated systems [20]. In the doped Mott system, the effective charges, like holes in underdoped cuprate, move on an inhomogeneous background with strong electronic correlation, exhibiting the VRH behavior of motion [21]. When measuring the specific heat down to $400 \mathrm{mK}$, a sizable residual coefficient can be observed, which is in sharp contrast to the strong insulatorlike behavior of the system. By applying pressure, a transition from insulator to metal occurs, and a superconducting transition also appears at about $T_{c}=5.1 \mathrm{~K}$. The zeroresistance temperature depends strongly on external magnetic field and applied current, suggesting a fragile superfluid density. Our results indicate that the superconductivity is established by inducing diluted Cooper pairs on top of the FeSe planes, which show a background of strong correlation.

\section{METHODS}

By using the solid-state reaction method, polycrystalline samples of $\mathrm{CsFe}_{4-x} \mathrm{Se}_{4}$ were successfully synthesized. First, FeSe precursors were prepared by reacting Fe powder and Se powder (Alfa Aesar, 99.999\% and 99.99\%, respectively) at $950 \mathrm{~K}$ for $24 \mathrm{~h}$ in sealed quartz tubes. Second, we reground the FeSe precursors, mixed them with a stoichiometric amount of alkali metal Cs (Alfa Aesar, 99.95\%) in an alumina crucible, and sealed the crucible into an evacuated quartz tube under vacuum. All manipulations were performed in a glove box filled with argon gas. The tube was subsequently heated up to and kept at $873 \mathrm{~K}$ for $24 \mathrm{~h}$. Then, the obtained sample was pulverized, pressed into a pellet, sealed in a quartz tube under vacuum, and heated up and kept at $923 \mathrm{~K}$ for $72 \mathrm{~h}$. Finally, dark-colored samples were obtained. The samples are sensitive to air, and thus they are usually vacuum packed and put in the glove box. The synthesis process is similar to that of previous work [18]. The only difference is that in previous work, the quartz tube was sealed with Ar gas, while in our experiments, the quartz tube is sealed under evacuation.

The x-ray-diffraction (XRD) data were obtained with a Bruker D8 Advanced diffractometer, and the Rietveld refinements [22] were conducted with the TOPAS4.2 software [23]. The energy dispersive spectrum (EDS) measurements were carried out with a Phenom ProX scanning electron microscope (SEM) at an accelerating voltage of $15 \mathrm{kV}$. The resistivity data at ambient pressure were measured on the Physical Property Measurement System (PPMS, Quantum Design). Gold wires with a diameter of 30 micrometers were attached to the sample with silver paint, forming a standard four-probe configuration, with a contact resistance of magnitude $1 \Omega$. The magnetization measurements were performed on a SQUID-VSM (Quantum Design). The specific heat was measured with a thermal-relaxation method using an option of the PPMS with a $\mathrm{He}^{3}$ insert, which allowed us to measure specific heat down to $0.4 \mathrm{~K}$. The resistivity data under high pressure were obtained by using a diamond-anvil-cell (DAC) module (cryoDAC-PPMS, Almax easyLab). The Pt electrodes were attached to the sample with a four-probe van der Pauw method [24], and the contact resistance had a magnitude of $1 \Omega$ above $1 \mathrm{GPa}$. The pressure medium used in the DAC was a fine powder of $\mathrm{NaCl}$, and the gasket was made of T301 stainless steel. The applied pressure was calculated by measuring the shift of the ruby $\mathrm{R}_{1}$ luminescent line [25].

\section{RESULTS}

Comparing with the closely related structure $\mathrm{Cs}_{1-x} \mathrm{Fe}_{2-y} \mathrm{Se}_{2}$ (for simplicity, we denote it as $\mathrm{CsFe}_{2} \mathrm{Se}_{2}$ hereafter), the new compound $\mathrm{CsFe}_{4-x} \mathrm{Se}_{4}$ naturally possesses the orthorhombic symmetry at room temperature. Schematic structures of these two compounds are shown in Fig. 1(a). The crystal structure of $\mathrm{CsFe}_{2} \mathrm{Se}_{2}$ is also plotted in order to have a close comparison with that of $\mathrm{CsFe}_{4-x} \mathrm{Se}_{4}$. The Cs plane in the middle of the structure of $\mathrm{CsFe}_{2} \mathrm{Se}_{2}$ is replaced by the Cs one-dimensional chains in $\mathrm{CsFe}_{4-x} \mathrm{Se}_{4}$ so that $\mathrm{CsFe}_{4-x} \mathrm{Se}_{4}$ possesses the $\mathrm{C} 2$ symmetry instead of the $\mathrm{C} 4$ symmetry; this difference is shown directly in Fig. 1(b) as the top views of the Cs structures for the two systems. The XRD pattern with the Rietveld refinement [22] [Fig. 1(c)] and the SEM images with EDS [Fig. 1(d)] are measured to verify the phase of our sample. The Rietveld refinements are carried out with the TOPAS4.2 software [23]. From the refinements, we can obtain the agreement factors $R_{w p}=4.69 \%$ and $R_{p}=$ $3.70 \%$, and the relatively small values mean that the calculated profile agrees with our experimental data quite well. The lattice parameters determined here are $a=$ 5.45(2) $\AA$, $b=5.46(0) \AA$, and $c=15.62(8) \AA$, which are close to those of previous work [18]. The inset gives the details of the XRD data in the low-angle region, which shows the typical peaks owing to the $\mathrm{C} 2$ symmetry. The phase indices marked with black vertical lines are calculated by the $\mathrm{CsFe}_{2} \mathrm{Se}_{2}$ phase with the converted lattice parameter $(a=b=3.852 \AA, c=15.628 \AA)$, while the red indices belong to $\mathrm{CsFe}_{4-x} \mathrm{Se}_{4}$. Here, the black vertical lines show the coincident positions of $\mathrm{CsFe}_{2} \mathrm{Se}_{2}$ and $\mathrm{CsFe}_{4-x} \mathrm{Se}_{4}$, which does not mean the existence of $\mathrm{CsFe}_{2} \mathrm{Se}_{2}$. A tiny peak at about 45 degrees comes from the impurity of iron, and the atomic ratio is about $0.6 \%$, which has been marked in Fig. 1(c). Thus, our sample is of good quality and does not contain the impurity phase like its precursor FeSe. We determine the occupancies of $\mathrm{Fe}$ and $\mathrm{Cs}$ by performing Rietveld refinement of the XRD data, and the obtained occupancies of $\mathrm{Fe}$ and $\mathrm{Cs}$ are 0.908 and 1.045, respectively. Meanwhile, we use the x-ray energy dispersive spectrum to analyze the compositions of $\mathrm{Cs}, \mathrm{Fe}$, and $\mathrm{Se}$ in the samples (for details, please refer to Sec. II). The mean 
(a)
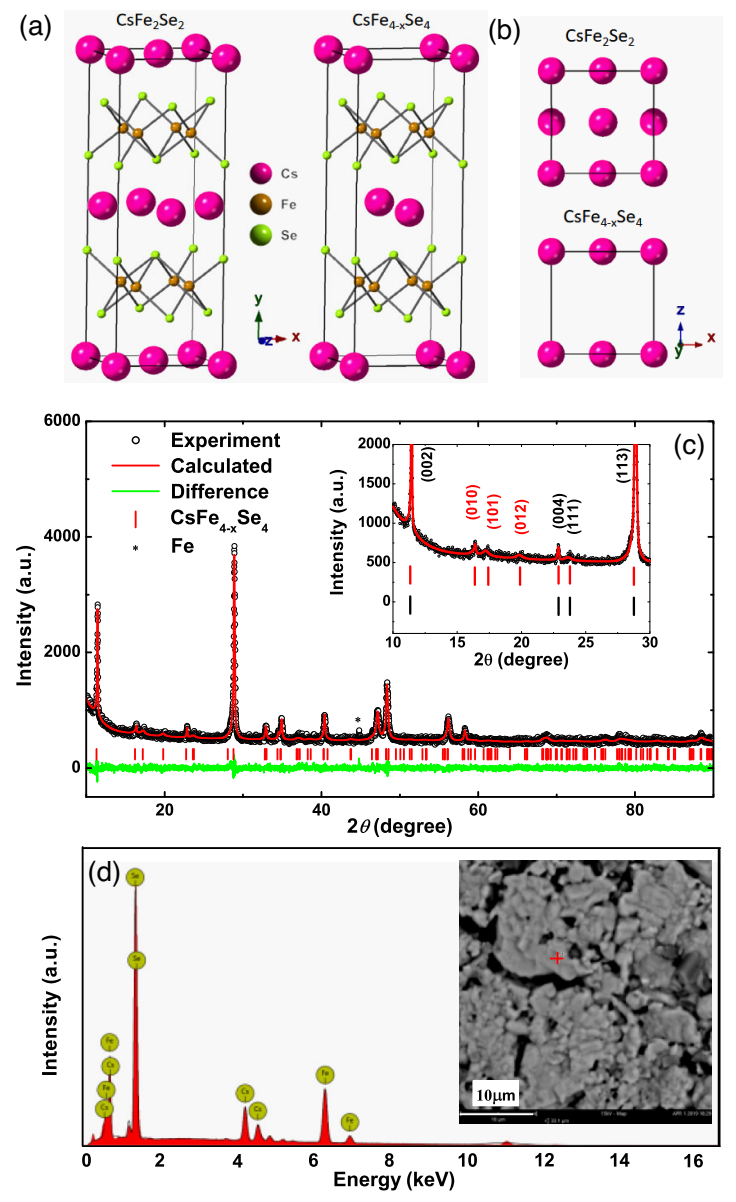

FIG. 1. Structure and compositional analysis of $\mathrm{CsFe}_{4-x} \mathrm{Se}_{4}$. (a) Schematic crystal structure of $\mathrm{CsFe}_{2} \mathrm{Se}_{2}$ and $\mathrm{CsFe}_{4-x} \mathrm{Se}_{4}$. (b) Crystal structure of $\mathrm{CsFe}_{2} \mathrm{Se}_{2}$ (restructured) and $\mathrm{CsFe}_{4-x} \mathrm{Se}_{4}$ viewed along the [001] direction. (c) X-ray-diffraction pattern for polycrystalline $\mathrm{CsFe}_{4-x} \mathrm{Se}_{4}$ with the Rietveld refinement. The inset in panel (c) shows typical peaks of $\mathrm{CsFe}_{4-x} \mathrm{Se}_{4}$, marked with red indices. The black vertical lines show the coincident indices of $\mathrm{CsFe}_{2} \mathrm{Se}_{2}$ and $\mathrm{CsFe}_{4-x} \mathrm{Se}_{4}$. (d) Typical energy dispersive spectrum, which shows the compositional ratio of the measured spot. The averaged composition of $\mathrm{Cs}: \mathrm{Fe}: \mathrm{Se}=1.2: 3.95: 4$ was obtained by the measurements at 19 spots and areas. The inset shows a scanning electron micrograph image.

value of the ratio of the three elements is roughly $\mathrm{Cs}: \mathrm{Fe}: \mathrm{Se}=1.2: 3.95: 4$, which means our samples have a little excess $\mathrm{Cs}$ and small amounts of $\mathrm{Fe}$ vacancies compared with the stoichiometric standard formula. The existence of Fe vacancy seems to be a common feature in the intercalated FeSe systems $[10,11,13]$; in addition, the excess Cs may exist in the form of Cs oxides because Cs is very active, and a weak reaction with a slight amount of residual oxygen may be inevitable during the synthesis process.

As mentioned before, the resistivity of the $\mathrm{CsFe}_{4-x} \mathrm{Se}_{4}$ polycrystalline sample behaves as an insulator, which is shown in Fig. 2(a). As shown in Fig. 2(b), the data between $20 \mathrm{~K}$ and $300 \mathrm{~K}$ are fitted roughly by the formula of threedimensional VRH [20], namely, $\rho=\rho_{0} \cdot \exp \left[\left(T_{0} / T\right)^{1 / 4}\right]$.
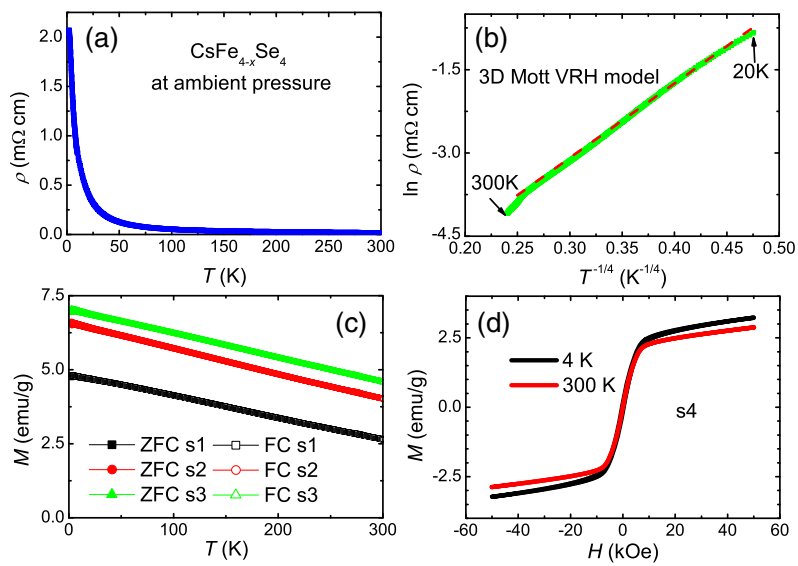

FIG. 2. Resistivity and magnetization of $\mathrm{CsFe}_{4-x} \mathrm{Se}_{4}$. (a) Temperature dependence of resistivity of $\mathrm{CsFe}_{4-x} \mathrm{Se}_{4}$ at ambient pressure. (b) Resistivity data from $20 \mathrm{~K}$ to $300 \mathrm{~K}$, showing a roughly linear behavior (red dashed line), which is consistent with the VRH model for the three-dimensional correlated systems. (c) Temperature dependence of magnetization measured at the field of $1 \mathrm{~T}$ for three samples. (d) Magnetic field dependence of magnetization measured at $4 \mathrm{~K}$ and $300 \mathrm{~K}$ for another sample.

Surprisingly, this relation roughly holds in a wide temperature region. The slight deviation below $20 \mathrm{~K}$ may indicate a possible change of the conduction mechanism or a modification to the 3D VRH model. We also try to fit the resistivity data with other possible models, such as the band gap model $\left[\rho=\rho_{0} \cdot \exp \left(T_{0} / T\right)\right], 1 \mathrm{D}\left[\rho=\rho_{0}\right.$. $\left.\exp \left[\left(T_{0} / T\right)^{1 / 2}\right]\right]$ and $2 \mathrm{D}\left[\rho=\rho_{0} \cdot \exp \left[\left(T_{0} / T\right)^{1 / 3}\right]\right] \mathrm{VRH}$ models $[20,26]$, and the small polaron hopping model [27] $\left[\rho=\rho_{0} T \cdot \exp \left(T_{0} / T\right)\right]$, but all fittings fail. One can see these model fittings in Fig. 1 of the Supplemental Material [28]. Indeed, insulating grain boundaries in granular samples would also contribute such an insulatorlike feature. However, we did not see such a secondary insulating phase (if they existed on the surface of grains) from XRD. If this insulatorlike behavior arises just from the scattering of the grain boundaries (supposing metallic grains), under a high pressure, such as several GPa, we believe that the electric conduction through the grain boundaries would no longer be insulatorlike.

The temperature dependence of magnetization measured for three samples at the magnetic field of $1 \mathrm{~T}$ in both zerofield-cooling (ZFC) and field-cooling (FC) modes is plotted in Fig. 2(c). It is easy to find that the magnetization follows a roughly linearly decreasing behavior with a wide temperature region, which certainly violates the Curie-Weiss law, and the reason for the strange linear temperature dependence of magnetization is still unknown. The difference of magnetization values (about 25\%) between different samples in Fig. 2(c) may be induced by the different content of $\mathrm{Fe}$ impurities. Although the amount of Fe impurity is tiny, due to its ferromagnetic feature of $\mathrm{Fe}$, this will lead to a 
different background of total signal of magnetization. The content of $\mathrm{Fe}$ impurity in different samples should be slightly different so that there would be a difference of magnetization values. A slight difference (about $2 \%$ at $2 \mathrm{~K}$ ) between the magnetizations measured in the $\mathrm{ZFC}$ and $\mathrm{FC}$ modes below about $20 \mathrm{~K}$ is observed. The reason for this small difference is still unknown and could be attributed to the tiny impurity phase with magnetic hysteresis. The small amount of a possible impurity phase cannot be distinguished from the XRD data. If this possible minority phase arises from the residual $\mathrm{CsFe}_{2} \mathrm{Se}_{2}$, according to the difference of the ZFC-FC magnetizations, we can roughly estimate the ratio of the $\mathrm{CsFe}_{2} \mathrm{Se}_{2}$, which is less than $1 \mathrm{wt} \%$. We do not see any resistivity, and the magnetization drops in the low-temperature region on the insulating background at ambient pressure, which may also exclude the presence of $\mathrm{CsFe}_{2} \mathrm{Se}_{2}$ and $\mathrm{FeSe}$ since otherwise the resistivity and magnetization would show associated drops at corresponding temperatures. In addition, we measure the lowtemperature $M(T)$ curves at $H=10$ Oe with $\mathrm{ZFC}$ and FC modes. The data are shown in Fig. 2 of Ref. [28], which confirms the absence of a superconducting phase in asprepared $\mathrm{CsFe}_{4-x} \mathrm{Se}_{4}$ at ambient pressure. From the $\mathrm{M}(\mathrm{H})$ curves in Fig. 2(d), we can see a weak ferromagnetic behavior. We regard this signal as weak because the magnetic susceptibility with an external field of $1 \mathrm{~T}$ at $4 \mathrm{~K}$ is only about $3.4 \times 10^{-3} \mathrm{emu} \mathrm{cm}^{-3} \mathrm{Oe}^{-1}$, which is much smaller than that in normal ferromagnetic materials (about $1-10^{5} \mathrm{emu} \mathrm{cm}^{-3} \mathrm{Oe}^{-1}$ ). Thus, it is reasonable to attribute this weak ferromagnetic signal to the Fe impurity, which also consists of the XRD data and the values of magnetization in $M(T)$ curves shown in Fig. 2(c).

From our data, we may exclude the antiferromagnetic or ferromagnetic order below $300 \mathrm{~K}$ since we did not see any transition on the temperature dependence of magnetic susceptibility. From the magnitude of magnetization under $1 \mathrm{~T}$ (at least 100 times smaller than that in normal ferromagnetic materials) and the near coincidence of the ZFC and FC magnetizations, we can rule out the presence of ferromagnetic order above $300 \mathrm{~K}$. The coincidence of FC and $\mathrm{ZFC}$ magnetizations may also allow us to exclude the glassy magnetism. We could not exclude the existence of antiferromagnetic order above $300 \mathrm{~K}$ since our measurement was only done up to $300 \mathrm{~K}$. However, according to the data in previous work [18], the magnetic susceptibility does not show any peak or kink feature below $800 \mathrm{~K}$, so the antiferromagnetic order at high temperatures may also be excluded.

In order to unravel the puzzling insulatorlike behavior of $\mathrm{CsFe}_{4-x} \mathrm{Se}_{4}$, we carry out specific heat measurements. Considering the contribution of phonons and conducting electrons in a metal, the specific heat at low temperatures can be described by the Debye model, namely, $C / T=\gamma_{0}+\beta T^{2}+\delta T^{4}+\ldots$ Here, $\gamma_{0}$ is the specific heat coefficient, and $\beta$ and $\delta$ are temperature-independent fitting
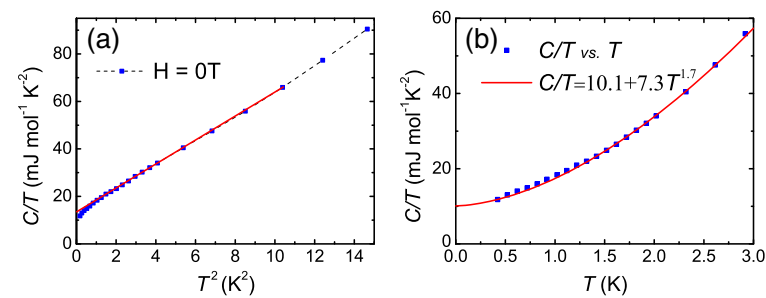

FIG. 3. Low-temperature specific heat of $\mathrm{CsFe}_{4-x} \mathrm{Se}_{4}$. (a) Quadratic temperature dependence of the specific heat coefficient $C / T$ measured down to $0.4 \mathrm{~K}$. The linear red curve gives the Debye model fit in the temperature range of 1.5 to $4 \mathrm{~K}$, which yields a residual coefficient of about $13.6 \mathrm{~mJ} / \mathrm{mol}^{-\mathrm{K}^{2}}$. (b) Temperature dependence of the specific heat coefficient $C / T$ in the temperature region from $0.4 \mathrm{~K}$ to $3 \mathrm{~K}$. The red curve gives a fitting with the formula $C / T=10.1+7.3 T^{1.7}\left(\mathrm{~mJ} / \mathrm{mol}^{-\mathrm{K}^{2}}\right)$. A sizable residual specific heat coefficient can be observed.

parameters. There should be no residual term $\gamma_{0}$ in the above description in the zero-temperature limit for a band insulator since this Somerfield term $\gamma_{0}$ reflects the finite quasiparticle DOS at the Fermi energy, which is zero in the band insulator. The terms with higher powers of temperature $\left(\delta T^{4}+\ldots\right)$ should be negligible in the low-temperature region since, in the formula of the Debye model for the phonon contribution to specific heat, the temperature is normalized by the Debye temperature $\Theta_{D}$, which takes the value of about $147.8 \mathrm{~K}$ (see below). In Fig. 3, we present the specific heat data of $\mathrm{CsFe}_{4-x} \mathrm{Se}_{4}$ down to $0.4 \mathrm{~K}$. Figure 3(a) shows the data $C / T$ versus $T^{2}$. One can see two anomalous and intriguing features here. First, the data do not satisfy the simple description of the Debye model. At temperatures above about $1.5 \mathrm{~K}$, one can see a roughly linear behavior of $C / T$ versus $T^{2}$. The red curve shows the Debye model fitting, which yields $\beta=$ $5.4 \mathrm{~mJ} /\left(\mathrm{mol}-\mathrm{K}^{4}\right)$. According to the Debye model, $\Theta_{D}=\left(12 \pi^{4} k_{B} N_{A} Z / 5 \beta\right)^{1 / 3}$, where $N_{A}=6.02 \times 10^{23} / \mathrm{mol}$ is the Avogadro constant, and $Z$ is the number of atoms in one unit cell; here, $Z=9$ for $\mathrm{CsFe}_{4-x} \mathrm{Se}_{4}$. Using the obtained value of $\beta$, we get the Debye temperature $\Theta_{D} \approx 147.8 \mathrm{~K}$. Below about $1.5 \mathrm{~K}$, however, the curve of $C / T$ vs $T^{2}$ shows a slight decline, which clearly deviates from the description of the Debye model. Second, to our surprise, there is a residual term of the specific heat coefficient $\gamma_{0}$ in the zero-temperature limit concerning the strong insulatorlike state. If we follow the linear extrapolation of the Debye model, as highlighted by the red linear line in Fig. 3(a), we obtain a residual term $\gamma_{0}=13.6 \mathrm{~mJ} / \mathrm{mol}^{-\mathrm{K}^{2}}$ at $T=0 \mathrm{~K}$. This result is totally unexpected for a band insulator. Even if we follow the decreasing trend of $C / T$ in the low-temperature region, we can still see a sizable value of $\gamma_{0}$. In order to carry out the empirical relation of $C / T$ vs $T$ in the lowtemperature region, we show the raw data in the temperature region of 0.4 to $3 \mathrm{~K}$ in Fig. 3(b), and we fit it with an 
empirical relation $C / T=\gamma_{0}+a T^{n}$. The best fitting yields a relation $C / T=10.1+7.3 T^{1.7} \mathrm{~mJ} / \mathrm{mol}-\mathrm{K}^{2}$. Thus, a residual term $\gamma_{0}$ clearly exists. Concerning the strong insulatorlike behavior seen from the resistivity, this residual term of specific heat in the zero-temperature limit indicates a nontrivial origin. Since the residual term $\gamma_{0}$ is quite large, we cannot attribute it to any possible metallic impurities.

In some amorphous compounds, a linear specific heat term could be observed [29]; however, as shown by the XRD data of our sample, the dominant phase here is $\mathrm{CsFe}_{4-x} \mathrm{Se}_{4}$, with a well-formed crystalline structure that does not show any trace of amorphousness. In previous studies on the spin-glass state of CuMn alloys [30], a linear term of specific heat was also observed, together with a deviation from the Debye model; this was attributed to the extra hyperfine entropy contribution of spins in the spinglass state. In a more general point of view, it was proposed that the linear term of specific heat might exist for a disordered insulator or glassy state, as discussed by Anderson et al. [31] and Phillip [32]. This possibility can also be ruled out since no evidence of a spin-glass transition is observed in our samples. If there were a spinglass transition, there would be a difference between the ZFC and FC magnetizations below the transition temperature, and a peak of magnetic susceptibility would appear; however, these are not observed in our samples. Although we have a slight amount of Fe vacancies and excess Cs in the samples, but they do not exist as segregations, in this case, they should not contribute a linear term of specific heat. Furthermore, even though these $\mathrm{Fe}$ vacancies and excess Cs are in a disordered state in the sample, due to their very small amounts, they should not contribute such a large residual linear term of specific heat. One fact to corroborate this point is that the contents of Fe vacancies and excess Cs are different in our samples than that of Ref. [18], but the linear terms of specific heat are quite similar in magnitude. Thus, we believe that the existence of the linear term of specific heat is intrinsic for the system.

In some spin-liquid candidates, a residual term of specific heat exists, which is considered as the contribution of the quantum spin fluctuations of a spin liquid at zero temperature [33]. Thus, we intend to conclude that the linear term, together with a deviation from the Debye model, may be attributed to the nontrivial ground state of the $\mathrm{CsFe}_{4-x} \mathrm{Se}_{4}$. To illustrate whether the ground state is a spin liquid, we can combine the measurements of low-temperature specific heat and thermal conductivity. However, since the present sample is a polycrystalline one, the transport of any kind of supermagnons or quantum spin fluctuations will be hindered by the grain boundaries or the interface between the grains. Thus, it may not be conclusive even though the thermal conductivity experiment does not show a residual term of $\kappa_{0} / T$ in the low-temperature region. Such experiments on single crystals of $\mathrm{CsFe}_{4-x} \mathrm{Se}_{4}$ are highly desired.
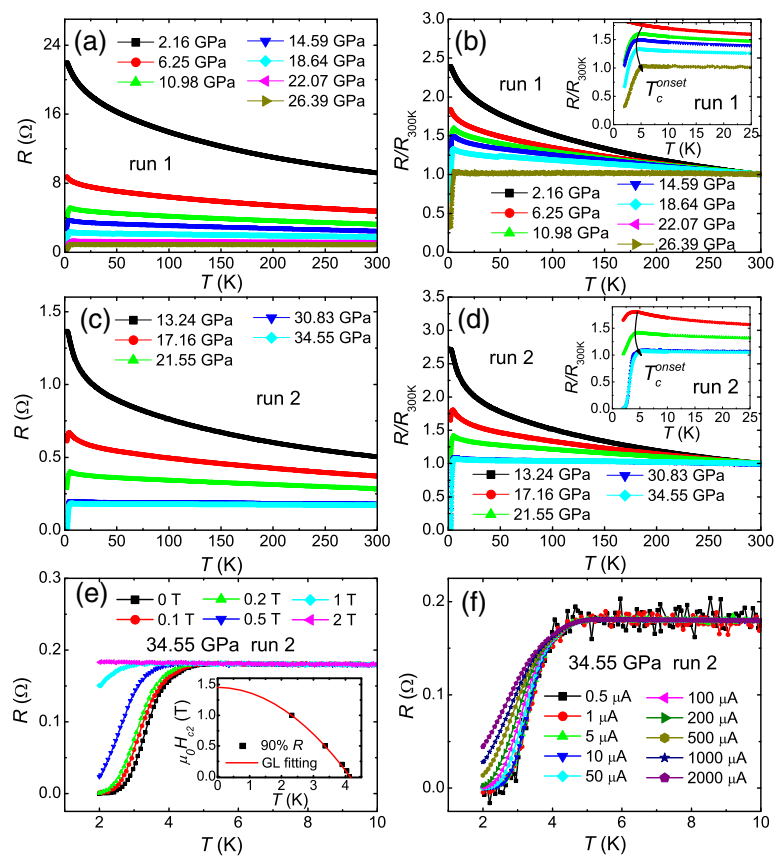

FIG. 4. Pressure-induced superconductivity in $\mathrm{CsFe}_{4-x} \mathrm{Se}_{4}$. (a,b) Temperature-dependent resistance and normalized resistance at high pressures for run 1. The inset in panel (b) shows an enlarged view on the normalized data in the low-temperature region $(0-25 \mathrm{~K})$. (c,d) Temperature-dependent resistance and normalized resistance at high pressures for run 2 . The inset in panel (d) shows an enlarged view of the same data in the lowtemperature region $(0-25 \mathrm{~K})$. (e) Superconducting transition curves measured under different magnetic fields at $34.55 \mathrm{GPa}$ of run 2. The inset in panel (e) shows the phase diagram of the upper critical field. (f) Superconductivity measured with different electric currents at $34.55 \mathrm{GPa}$ of run 2 .

As an effective and clean way to manipulate the properties of a compound, high pressure is also applied to this material. The results are shown in Figs. 4(a)-4(f). For the high-pressure measurements, we used different DACs for different runs of experiments. The culet size of DAC for run 1 [Figs. 4(a) and 4(b)] is $400 \mu \mathrm{m}$, while for run 2 [Figs. 4(c) and 4(d)], it is $300 \mu \mathrm{m}$; thus, the maximum pressure applied in run 2 is higher than that in run 1. By increasing the applied pressure, the insulatorlike behavior can be successively suppressed, and the system undergoes a transition from an insulator to a bad metal. The resistance decreases monotonously with applied pressure; meanwhile, the residual-resistivity-ratio $\left(R R R, R_{300 \mathrm{~K}} / R_{6 \mathrm{~K}}\right)$ increases from 0.42 to 0.96 in run 1 and from 0.39 to 0.93 in run 2. Accompanying the emergence of metallicity, superconductivity gradually appears. The resistivity starts to drop at about $5.1 \mathrm{~K}$ under a pressure of $10.98 \mathrm{GPa}$ in run 1 and 13.24 GPa in run 2. As the pressure keeps increasing, the $T_{c}^{\text {onset }}$ changes slightly, and the ratio of the superconducting phase becomes larger until zero resistance is measured at $30.83 \mathrm{GPa}$ in run 2 . The enlarged views of the data in the low-temperature region $(0-25 \mathrm{~K})$ are shown in the insets of 
Figs. 4(b) and 4(d), respectively. The low-temperature resistance data under different magnetic fields up to $2 \mathrm{~T}$ at $34.55 \mathrm{GPa}$ are also obtained and shown in Fig. 4(e). One can see that the magnetic field can easily suppress superconductivity. By taking $90 \%$ of the normal-state resistance as the criterion to determine $T_{c}$, we can obtain the phase diagram of the upper critical field $H_{c 2}(T)$, which is shown in the inset of Fig. 4(e). By fitting the data with the formula based on Ginzburg-Landau theory $H_{c 2}(T)=H_{c 2}(0)$. $\left[1-\left(T / T_{c}\right)^{2}\right]$, the $H_{c 2}(0)$ is about $1.45 \mathrm{~T}$, which is relatively small compared with that of other FeSe based superconductors.

One may argue that the superconductivity observed here may arise from some impurity phases. However, based on the obtained message of $T_{c}$ and $H_{c 2}(0)$, we can prove that the superconductivity observed here is induced by the main phase $\mathrm{CsFe}_{4-x} \mathrm{Se}_{4}$ rather than other impurity phases. Judging from the values of $T_{c}$, we can exclude the possibility that the superconducting phase comes from possible impurities like Fe [34], $\mathrm{Cs}_{0.8} \mathrm{Fe}_{2} \mathrm{Se}_{2}$ [35], or FeSe [2,4] since their $T_{c}$ values are very different from the observed $5.1 \mathrm{~K}$ here. To corroborate this, we emphasize that no clear trace of these impurity phases is visible from the $\mathrm{XRD}$ data. There is a concern that the superconductivity may come from the Se element, whose $T_{c}$ at high pressure is about $6.5 \mathrm{~K}$. We then measure the resistivity of elemental Se by applying pressure; the data are shown in Fig. 3 of Ref. [28]. Comparing with $\mathrm{Se}$ at a pressure of about $30 \mathrm{GPa}$, the $T_{c}$ of $\mathrm{CsFe}_{4-x} \mathrm{Se}_{4}$ is lower, and the phase lines of $H_{c 2}(T)$ are also different. Generally, the upper critical field $H_{c 2}(T)$ of the pressurized $\mathrm{CsFe}_{4-x} \mathrm{Se}_{4}$ is much lower than that of Se, and no trace of Se element can be observed from the XRD data. Thus, this possibility can also be ruled out. In addition, we want to emphasize that the appearance and disappearance of superconductivity in the present system follows very well in processes with increasing and decreasing pressure. After superconductivity appears at high pressure, when we lower the pressure, the superconductivity gradually disappears, and the strong insulating feature reappears. The data with decreasing pressure are shown in Fig. 4 of Ref. [28]. All these indicate that the superconductivity here is an intrinsic property of $\mathrm{CsFe}_{4-x} \mathrm{Se}_{4}$ under pressure. With these arguments and several rounds of control experiments, we can safely conclude that the superconductivity observed here is induced in the $\mathrm{CsFe}_{4-x} \mathrm{Se}_{4}$ system by a pressure effect.

The absence of zero resistance in runs 1 and 3 may be because we have used a relatively large measuring current $(1000 \mu \mathrm{A})$ concerning the very small sample size of the DAC. In Fig. 4(f), we show the superconducting transitions under different applied currents. Interestingly, we find that the superconducting transition can be easily affected by the applied current, although the onset temperature does not shift with the current. With a small measuring current, we can see the zero-resistance state; however, it is lost when the measuring current becomes large. We must emphasize that the vanishing of the zero-resistance state is not due to the heating effect since the onset transition temperature does not change at all with different currents. We could argue that this mimics the picture where the superconducting state is formed by the diluted superfluid density on top of the background of a correlated bad metal. The superconducting phenomenon can be repeatedly observed in each run of our high-pressure measurements. Since our sample is polycrystalline in nature, the effect of grain boundaries cannot be ignored. However, under such high pressures, the grain-boundary issue on the critical current density may not be serious for the following reasons. First, according to the Pippard relation $\xi=\hbar v_{F} / \Delta$, the low $T_{c}$ may correspond to a small gap and large coherence length, and the large coherence length will overcome the problem of grain boundaries. Second, the $\mathrm{CsFe}_{4-x} \mathrm{Se}_{4}$ system may not be anisotropic like many cuprates, and under high pressure, the weak-link effect is weakened; this process further weakens the influence of the grain boundaries on critical current density.

\section{DISCUSSION}

We have found several interesting features of the new compound $\mathrm{CsFe}_{4-x} \mathrm{Se}_{4}$. First, the material shows a strong insulatorlike behavior, which cannot be described by the model of a band insulator, while the temperature dependence of resistivity can be fitted to the 3D VRH model with correlations [20] in a wide temperature region. The DFT calculation shows that this compound should be a metal with the $3 d_{x z / y z / x y}$ orbits as the dominant ones [18]. In the first report about the properties of this material, the authors attributed this insulatorlike behavior to the Fe vacancy [18], which is unlikely since the Fe vacancy is only about $1.25 \%$ in our samples, according to our EDS analysis. Furthermore, given the presence of a certain amount of $\mathrm{Fe}$ vacancies, it is hard to understand why the hypothetical metallic background would show such a strong insulatorlike behavior. Second, we find that the specific heat shows a sizable residual coefficient $\gamma_{0}$, which is certainly unexpected for a band insulator. It is known that for a spin liquid, due to the existence of a quantum spin fluctuation at zero temperature, one expects a residual contribution to the specific heat coefficient. Thus, we believe that the residual linear specific heat contribution in the low-temperature region has a nontrivial origin; it is most likely related to the correlation effect in this compound. Third, we have observed superconductivity by suppressing this insulatorlike behavior after applying high pressure. From the upper critical field determined here, we find that $H_{c 2}(0)$ seems to be quite low. This case is different from other FeSe or FeAs based superconductors in which the upper critical field is generally quite high. More interestingly, we find that the zero-resistance superconducting temperature is dependent on the applied current, which reminds us that 
the superconducting state achieved under high pressure may be formed by the very diluted superfluid on the background of a correlated system. The same situation occurs in the underdoped cuprate superconductors in which the superfluid density is very low and the superconducting transition temperature is determined by this Bose-Einstein condensation temperature [36,37].

Actually, in the iron based superconductors, the correlation strength is orbital dependent and can be quite strong; in many cases, the $d_{x y}$ orbit shows a strong temperaturedependent correlation effect, called the orbital-selective Mottness [38]. Because of the complex charge-spin-lattice interactions, the correlation effect can be induced by the Hund's coupling effect with different strengths on different orbits, which may lead to the insulatorlike behavior [39]. Recently, the quasiparticle interference technique based on the scanning tunneling microscope measurements reveals strong and orbital-selective differences of quasiparticle weight $Z$ on all detectable bands over a wide energy range in bulk FeSe [40]. The authors conclude that orbitalselective strong correlations dominate the parent state of iron-based high-temperature superconductivity in FeSe; even the normal state looks like a metal. In the FeSe based family, there exist some other systems, such as the $\mathrm{BaFe}_{2} \mathrm{Se}_{3}$, which has been regarded as the orbital-selective Mott insulator [41]. Inelastic neutron scattering results show that this system has a block antiferromagnetic state with rather large magnetic moments [42]. They also tell us how the orbital degrees of freedom in iron-based compounds can help to stabilize an exotic magnetic state. By applying pressure, this Mott insulator turns gradually from a strong insulatorlike behavior to a metallic state, and finally superconductivity is observed [43]. Although the structures of $\mathrm{BaFe}_{2} \mathrm{Se}_{3}$ and the present system, $\mathrm{CsFe}_{4-x} \mathrm{Se}_{4}$, are different, the fundamental physics may be similar; namely, the orbital-selective Mottness can be suppressed by pressure, and charges then have more freedom to move and superconductivity is finally achieved. Superconductivity was also induced in another Fe-based compound, $\mathrm{FePSe}_{3}$, by pressure [44]. This material has a honeycomb structure at ambient pressure and undergoes a structural transition at high pressure. The authors attributed the emergence of superconductivity to the crossover of the spin states via the structural transition. An intuitive picture called the Gossamer state was given by Laughlin [45] and developed by Zhang et al. [46] to describe the unique superconducting state in cuprates, which postulates a diluted superfluid density on the correlated background. We think that this picture may also be applied to our present system. Our present study illustrates that the $\mathrm{CsFe}_{4-x} \mathrm{Se}_{4}$ system may provide a strong correlated electronic platform for inducing unconventional superconductivity. It calls for theoretical efforts beyond the DFT calculations to understand how strong the orbital-selective correlations are and how the
Mottness is established in this particular system, and finally why superconductivity is induced by applying pressure. In a newly found $\mathrm{Cu}$-based oxy-arsenide $\mathrm{RE}_{2} \mathrm{Cu}_{5} \mathrm{As}_{3} \mathrm{O}_{2}$ $(\mathrm{RE}=\mathrm{La}, \mathrm{Pr}, \mathrm{Nd})$ [47], it seems that the correlation effect is not very strong, and a Fermi liquid behavior appears in the normal state, which is different from the present system $\mathrm{CsFe}_{4-x} \mathrm{Se}_{4}$. However, in Fe based superconductors, most systems in the parent state show bad metal behavior depending on the subtle balance between itinerancy and localization of the $d$-orbital electrons. As far as we know, besides $\mathrm{BaFe}_{2} \mathrm{Se}_{3}, \mathrm{CsFe}_{4-x} \mathrm{Se}_{4}$ is another one showing a strong correlation effect, and superconductivity is induced by pressurizing the parent state with Mottness, which is similar to the cuprates in this regard. However, compared to $\mathrm{BaFe}_{2} \mathrm{Se}_{3}, \mathrm{CsFe}_{4-x} \mathrm{Se}_{4}$ seems to be more fundamental in structure because it possesses the basic FeSe layers.

\section{CONCLUSIONS}

We successfully synthesize the newly discovered FeSe-based compound $\mathrm{CsFe}_{4-x} \mathrm{Se}_{4}$ and find several intriguing physical proprieties. At ambient pressure, the temperature-dependent resistivity shows insulatorlike behavior and can be described by the 3D VRH model for correlated systems. The temperature dependence of magnetization does not obey the Curie-Weiss law but shows a unique linear behavior with a negative slope versus temperature in a wide temperature region. The specific heat down to $400 \mathrm{mK}$ shows a sizable residual specific heat coefficient, which is unexpected for a band insulator. We intend to attribute this residual specific heat coefficient to the quantum spin fluctuations of the system in the zero-temperature limit, and the background is argued to be a Mott insulator. By applying high pressure, the insulatorlike behavior is gradually suppressed, and superconductivity appears at about $5.1 \mathrm{~K}$. The superconductivity can be suppressed both by an external magnetic field and electric current, which suggests that the superfluid density is fragile in this system. We believe that $\mathrm{CsFe}_{4-x} \mathrm{Se}_{4}$ can provide a platform to explore unconventional superconductivity established by diluted Cooper pairs on top of a strong correlation background.

\section{ACKNOWLEDGMENTS}

We are grateful to Louis Taillefer and Shiyan Li for discussions and the preliminary measurements of lowtemperature thermal conductivity. This work was supported by the National Key R\&D Program of China (Grants No. 2016YFA0300401 and No. 2016YFA0401704) and National Natural Science Foundation of China (Grants No. A0402/11534005, No. A0402/11927809, No. A0402/ 13001167, and No. A0402/11674164). 
[1] F.-C. Hsu, J.-Y. Luo, K.-W. Yeh, T.-K. Chen, T.-W. Huang, P. M. Wu, Y.-C. Lee, Y.-L. Huang, Y.-Y. Chu, D.-C. Yan, and M.-K. Wu, Superconductivity in the PbO-Type Structure $\alpha$-FeSe, Proc. Natl. Acad. Sci. U.S.A. 105, 14262 (2008).

[2] S. Medvedev, T. M. McQueen, I. A. Troyan, T. Palasyuk, M. I. Eremets, R. J. Cava, S. Naghavi, F. Casper, V. Ksenofontov, G. Wortmann, and C. Felser, Electronic and Magnetic Phase Diagram of $\beta-\mathrm{Fe}_{1.01}$ Se with Superconductivity at $36.7 \mathrm{~K}$ under Pressure, Nat. Mater. 8, 630 (2009).

[3] T. Imai, K. Ahilan, F. L. Ning, T. M. McQueen, and R. J. Cava, Why Does Undoped FeSe Become a High- $\mathrm{T}_{c}$ Superconductor under Pressure, Phys. Rev. Lett. 102, 177005 (2009).

[4] A. I. Coldea and M. D. Watson, The Key Ingredients of the Electronic Structure of FeSe, Annu. Rev. Condens. Matter Phys. 9, 125 (2018).

[5] Q.-Y. Wang, Z. Li, W.-H. Zhang, Z.-C. Zhang, J.-S. Zhang, W. Li, H. Ding, Y.-B. Ou, P. Deng, K. Chang, J. Wen, C.-L. Song, K. He, J.-F. Jia, S.-H. Ji, Y.-Y. Wang, L.-L. Wang, X. Chen, X.-C. Ma, and Q.-K. Xue, Interface-Induced HighTemperature Superconductivity in Single Unit-Cell FeSe Films on $\mathrm{SrTiO}_{3}$, Chin. Phys. Lett. 29, 037402 (2012).

[6] S. He, J. He, W. Zhang, L. Zhao, D. Liu, X. Liu, D. Mou, Y.-B. Ou, Q.-Y. Wang, Z. Li, L. Wang, Y. Peng, Y. Liu, C. Chen, L. Yu, G. Liu, X. Dong, J. Zhang, C. Chen, Z. Xu, X. Chen, X. Ma, Q. Xue, and X. J. Zhou, Phase Diagram and Electronic Indication of High-Temperature Superconductivity at $65 \mathrm{~K}$ in Single-Layer FeSe Films, Nat. Mater. 12, 605 (2013).

[7] Z. Zhang, Y.-H. Wang, Q. Song, C. Liu, R. Peng, K. A. Moler, D. Feng, and Y. Wang, Onset of the Meissner Effect at $65 \mathrm{~K}$ in FeSe Thin Film Grown on $\mathrm{Nb}$-Doped $\mathrm{SrTiO}_{3}$ Substrate, Sci. Bull. 60, 1301 (2015).

[8] J. Guo, S. Jin, G. Wang, S. Wang, K. Zhu, T. Zhou, M. He, and $\mathrm{X}$. Chen, Superconductivity in the Iron Selenide $\mathrm{K}_{x} \mathrm{Fe}_{2} \mathrm{Se}_{2} \quad(0 \leq x \leq 1.0)$, Phys. Rev. B 82, 180520(R) (2010).

[9] T. P. Ying, X. L. Chen, G. Wang, S. F. Jin, T. T. Zhou, X. F. Lai, H. Zhang, and W. Y. Wang, Observation of Superconductivity at $30 \sim 46 \mathrm{~K}$ in $\mathrm{A}_{x} \mathrm{Fe}_{2} \mathrm{Se}_{2}(\mathrm{~A}=\mathrm{Li}, \mathrm{Na}, \mathrm{Ba}, \mathrm{Sr}$, $\mathrm{Ca}, \mathrm{Yb}$, and $\mathrm{Eu}$ ), Sci. Rep. 2, 426 (2012).

[10] C.-H. Wang, C.-C. Lee, G.-T. Huang, J.-Y. Yang, M.-J. Wang, H.-S. Sheu, J.-J. Lee, and M.-K. Wu, Role of the Extra Fe in $\mathrm{K}_{2-x} \mathrm{Fe}_{4+y} \mathrm{Se}_{5}$ Superconductors, Proc. Natl. Acad. Sci. U.S.A. 116, 1104 (2019).

[11] A. F. Wang, J. J. Ying, Y. J. Yan, R. H. Liu, X. G. Luo, Z. Y. Li, X. F. Wang, M. Zhang, G. J. Ye, P. Cheng, Z. J. Xiang, and X.H. Chen, Superconductivity at $32 \mathrm{~K}$ in SingleCrystalline $\mathrm{Rb}_{x} \mathrm{Fe}_{2-y} \mathrm{Se}_{2}$, Phys. Rev. B 83, 060512(R) (2011).

[12] A. Krzton-Maziopa, Z. Shermadini, E. Pomjakushina, V. Pomjakushin, M. Bendele, A. Amato, R. Khasanov, H. Luetkens, and K. Conder, Synthesis and Crystal Growth of $\mathrm{Cs}_{0.8}\left(\mathrm{FeSe}_{0.98}\right)_{2}$ : A New Iron-Based Superconductor with $\mathrm{T}_{c}=27 \mathrm{~K}$, J. Phys. Condens. Matter 23, 052203 (2011).

[13] H.-D. Wang, C.-H. Dong, Z.-J. Li, Q.-H. Mao, S.-S. Zhu, C.-M. Feng, H. Q. Yuan, and M.-H. Fang, Superconductivity at $32 \mathrm{~K}$ and Anisotropy in $\mathrm{Tl}_{0.58} \mathrm{Rb}_{0.42} \mathrm{Fe}_{1.72} \mathrm{Se}_{2}$ Crystals, Europhys. Lett. 93, 47004 (2011).

[14] M. H. Fang, H. D. Wang, C. H. Dong, Z. J. Li, C. M. Feng, J. Chen, and H. Q. Yuan, Fe-Based Superconductivity with $\mathrm{T}_{c}=31 \mathrm{~K}$ Bordering an Antiferromagnetic Insulator in (TI, K)Fe $\mathrm{Fe}_{x} \mathrm{Se}_{2}$, Europhys. Lett. 94, 27009 (2011).

[15] D. P. Shoemaker, D. Y. Chung, H. Claus, M. C. Francisco, S. Avci, A. Llobet, and M. G. Kanatzidis, Phase Relations in $\mathrm{K}_{x} \mathrm{Fe}_{2-y} \mathrm{Se}_{2}$ and the Structure of Superconducting $\mathrm{K}_{x} \mathrm{Fe}_{2} \mathrm{Se}_{2}$ via High-Resolution Synchrotron Diffraction, Phys. Rev. B 86, 184511 (2012).

[16] X. F. Lu, N. Z. Wang, H. Wu, Y. P. Wu, D. Zhao, X. Z. Zeng, X. G. Luo, T. Wu, W. Bao, G. H. Zhang, F. Q. Huang, Q. Z. Huang, and X. H. Chen, Coexistence of Superconductivity and Antiferromagnetism in $\left(\mathrm{Li}_{0.8} \mathrm{Fe}_{0.2}\right) \mathrm{OHFeSe}$, Nat. Mater. 14, 325 (2015).

[17] R. Sun, S. Jin, L. Gu, Q. Zhang, Q. Huang, T. Ying, Y. Peng, J. Deng, Z. Yin, and X. Chen, Intercalating Anions between Terminated Anion Layers: Unusual Ionic S-Se Bonds and Hole-Doping Induced Superconductivity in $\mathrm{S}_{0.24}\left(\mathrm{NH}_{3}\right)_{0.26} \mathrm{Fe}_{2} \mathrm{Se}_{2}$, J. Am. Chem. Soc. 141, 13849 (2019).

[18] K. Li, Q.-Z. Huang, Q. Zhang, Z. Xiao, T. Kamiya, H. Hosono, D. Yuan, J. Guo, and X. Chen, $\mathrm{CsFe}_{4-\delta} \mathrm{Se}_{4}: A$ Compound Closely Related to Alkali-Intercalated FeSe Superconductors, Inorg. Chem. 57, 4502 (2018).

[19] S. Kasahara, T. Watashige, T. Hanaguri, Y. Kohsaka, T. Yamashita, Y. Shimoyama, Y. Mizukami, R. Endo, H. Ikeda, K. Aoyama, T. Terashima, S. Uji, T. Wolf, H. von Löhneysen, T. Shibauchi, and Y. Matsuda, Field-Induced Superconducting Phase of FeSe in the BCS-BEC CrossOver, Proc. Natl. Acad. Sci. U.S.A. 111, 16309 (2014).

[20] N. F. Mott, Conduction in Non-crystalline Materials, Philos. Mag. 19, 835 (1969).

[21] F. Abdel-Wahab and K. Shimakawa, A Percolation Approach to Variable Range Hopping in High $\mathrm{T}_{c}$ Superconducting Cuprates, Philos. Mag. Lett. 71, 351 (1995).

[22] H. M. Rietveld, A Profile Refinement Method for Nuclear and Magnetic Structures, J. Appl. Crystallogr. 2, 65 (1969).

[23] R. W. Cheary and A. Coelho, A Fundamental Parameters Approach to X-Ray Line-Profile Fitting, J. Appl. Crystallogr. 25, 109 (1992).

[24] L. J. van der Pauw, A Method of Measuring Specific Resistivity and Hall Effect of Discs of Arbitrary Shape, Philips Res. Rep. 13, 1 (1958).

[25] H. K. Mao, J. Xu, and P. M. Bell, Calibration of the Ruby Pressure Gauge to 800 kbar under Quasi-Hydrostatic Conditions, J. Geophys. Res. Solid Earth 91, 4673 (1986).

[26] H.-C. Wu, A. N. Chaika, M.-C. Hsu, T.-W. Huang, M. Abid, M. Abid, V. Yu. Aristov, O. V. Molodtsova, S. V. Babenkov, Y. Niu, B. E. Murphy, S. A. Krasnikov, O. Lübben, H. Liu, B. S. Chun, Y. T. Janabi, S. N. Molotkov, I. V. Shvets, A. I. Lichtenstein, M. I. Katsnelson, and C.-R. Chang, Large Positive In-Plane Magnetoresistance Induced by Localized States at Nanodomain Boundaries in Graphene, Nat. Commun. 8, 14453 (2017).

[27] R. Schmidt, A. Basu, and A. W. Brinkman, Small Polaron Hopping in Spinel Manganates, Phys. Rev. B 72, 115101 (2005). 
[28] See Supplemental Material at http://link.aps.org/ supplemental/10.1103/PhysRevX.10.041008 for extra data of fittings of resistivity with other models, low field ZFC and FC magnetizations, comparison of superconducting transitions between Se and present sample, and superconducting transitions in the decreasing pressure process.

[29] M. A. Ramos, C. Talón, R. J. Jiménez-Riobóo, and S. Vieira, Low-Temperature Specific Heat of Structural and Orientational Glasses of Simple Alcohols, J. Phys. Condens. Matter 15, S1007 (2003).

[30] D. L. Martin, Specific Heat of Spin-Glass CuMn below $3 \mathrm{~K}$, Phys. Rev. B 20, 368 (1979).

[31] P. W. Anderson, B. I. Halperin, and C. M. Varma, Anomalous Low Temperature Thermal Properties of Glasses and Spin Glasses, Phil. Mag. 25, 1 (1972).

[32] W. A. Phillips, Tunneling States in Amorphous Solids, J. Low Temp. Phys. 7, 351 (1972).

[33] O. Mustonen, S. Vasala, E. Sadrollahi, K. P. Schmidt, C. Baines, H. C. Walker, I. Terasaki, F. J. Litterst, E. BaggioSaitovitch, and M. Karppinen, Spin-Liquid-like State in a Spin-1/2 Square-Lattice Antiferromagnet Perovskite Induced by $d^{10}-d^{0}$ Cation Mixing, Nat. Commun. 9, 1085 (2018).

[34] K. Shimizu, D. Takao, S. Furomoto, and K. Amaya, Pressure-Induced Superconductivity in Li and Fe, Physica C 408-410, 750 (2004).

[35] G. Seyfarth, D. Jaccard, P. Pedrazzini, A. Krzton-Maziopa, E. Pomjakushina, K. Conder, and Z. Shermadini, Pressure Cycle of Superconducting $\mathrm{Cs}_{0.8} \mathrm{Fe}_{2} \mathrm{Se}_{2}$ : A Transport Study, Solid State Commun. 151, 747 (2011).

[36] G. Deutscher, Coherence and Single-Particle Excitations in the High-Temperature Superconductors, Nature (London) 397, 410 (1999).

[37] Z. A. Xu, N. P. Ong, Y. Wang, T. Kakeshita, and S. Uchida, Vortex-like Excitations and the Onset of Superconducting Phase Fluctuation in Underdoped $\mathrm{La}_{2-x} \mathrm{Sr}_{x} \mathrm{CuO}_{4}$, Nature (London) 406, 486 (2000).
[38] R. Yu and Q. Si, Orbital-Selective Mott Phase in Multiorbital Models for Alkaline Iron Selenides $\mathrm{K}_{1-x} \mathrm{Fe}_{2-y} \mathrm{Se}_{2}$, Phys. Rev. Lett. 110, 146402 (2013).

[39] Z. P. Yin, K. Haule, and G. Kotliar, Kinetic Frustration and the Nature of the Magnetic and Paramagnetic States in Iron Pnictides and Iron Chalcogenides, Nat. Mater. 10, 932 (2011).

[40] A. Kostin, P. O. Sprau, A. Kreisel, Y. X. Chong, A. E. Böhmer, P. C. Canfield, P. J. Hirschfeld, B. M. Andersen, and J. C. S. Davis, Imaging Orbital-Selective Quasiparticles in the Hund's Metal State of FeSe, Nat. Mater. 17, 869 (2018).

[41] J. Rincón, A. Moreo, G. Alvarez, and E. Dagotto, Exotic Magnetic Order in the Orbital-Selective Mott Regime of Multiorbital Systems, Phys. Rev. Lett. 112, 106405 (2014).

[42] M. Mourigal, S. Wu, M. B. Stone, J. R. Neilson, J. M. Caron, T. M. McQueen, and C. L. Broholm, Block Magnetic Excitations in the Orbitally Selective Mott Insulator $\mathrm{BaFe}_{2} \mathrm{Se}_{3}$, Phys. Rev. Lett. 115, 047401 (2015).

[43] J. Ying, H. Lei, C. Petrovic, Y. Xiao, and V. V. Struzhkin, Interplay of Magnetism and Superconductivity in the Compressed Fe-Ladder Compound $\mathrm{BaFe}_{2} \mathrm{Se}_{3}$, Phys. Rev. B 95, 241109(R) (2017).

[44] Y. Wang, J. Ying, Z. Zhou, J. Sun, T. Wen, Y. Zhou, N. Li, Q. Zhang, F. Han, Y. Xiao, P. Chow, W. Yang, V. V. Struzhkin, Y. Zhao, and H.-K. Mao, Emergent Superconductivity in an Iron-Based Honeycomb Lattice Initiated by Pressure-Driven Spin-Crossover, Nat. Commun. 9, 1914 (2018).

[45] R. B. Laughlin, Gossamer Superconductivity, arXiv:condmat/0209269v1.

[46] F. C. Zhang, Gossamer Superconductor, Mott Insulator, and Resonating Valence Bond State in Correlated Electron Systems, Phys. Rev. Lett. 90, 207002 (2003).

[47] X. Chen, J. G. Guo, C. S. Gong, E. J. Cheng, C. C. Le, N. Liu, T. P. Ying, Q. H. Zhang, J. P. Hu, S. Y. Li, and X. L. Chen, Anomalous Dome-like Superconductivity in $\mathrm{RE}_{2}\left(\mathrm{Cu}_{1-x} \mathrm{Ni}_{x}\right)_{5} \mathrm{As}_{3} \mathrm{O}_{2}(\mathrm{RE}=\mathrm{La}, \operatorname{Pr}, \mathrm{Nd})$, iScience 14, 171 (2019). 